\title{
Critical Practices in Rigorously Assessing the Inherent Activity of Nanoparticle Electrocatalysts
}

Sean T. Dix ${ }^{1,2}$, Shawn Lu ${ }^{1,2}$, Suljo Linic ${ }^{1,2, *}$

${ }^{1}$ Department of Chemical Engineering, University of Michigan, Ann Arbor, MI 48109, USA.

${ }^{2}$ Michigan Catalysis Science and Technology Institute (MiCSTI), University of Michigan, Ann Arbor, MI 48109, USA.

*Corresponding Author. Email: linic@umich.edu 


\section{Oxygen Reduction Kinetic Data}

To generate the plots showing the effects of Thiele modulus on specific activity, experimental Pt catalyzed ORR data was used. This data was obtained by using a planar Pt-poly (Pine) fixed electrode in a traditional 3-electrode RDE setup. An in-house built all Teflon cell housed the reference electrode $(\mathrm{Hg} / \mathrm{HgO}$, Pine), counter electrode ( $\mathrm{Pt}$ wire, Fisher) and the $0.1 \mathrm{M} \mathrm{NaOH}(30$ $w t \%$, Fisher). The reference electrode was calibrated against the reversible hydrogen electrode (RHE) in $\mathrm{H}_{2}$ (UHPG) saturated electrolyte before ORR voltammograms were measured. Surface area was measured in $\operatorname{Ar}$ (UHPG) saturated electrolyte by integrating the H-UPD potential region (0.05-0.4 V vs. RHE) of the Pt surface using a CO-poisoned baseline further described in the main text ${ }^{1}$. The solution resistance was measured to be $40 \mathrm{ohms}$ using hybrid electrochemical impedance spectroscopy. ORR measurements were done in $\mathrm{O}_{2}$ (UHPG) saturated electrolyte which was purged with gas for at least 20 minutes. As described in the main text, kinetic currents were decoupled from the linear sweep voltammograms using the Koutecky-Levich equation at 1600 RPM to produce the Tafel plot shown in Figure 1.

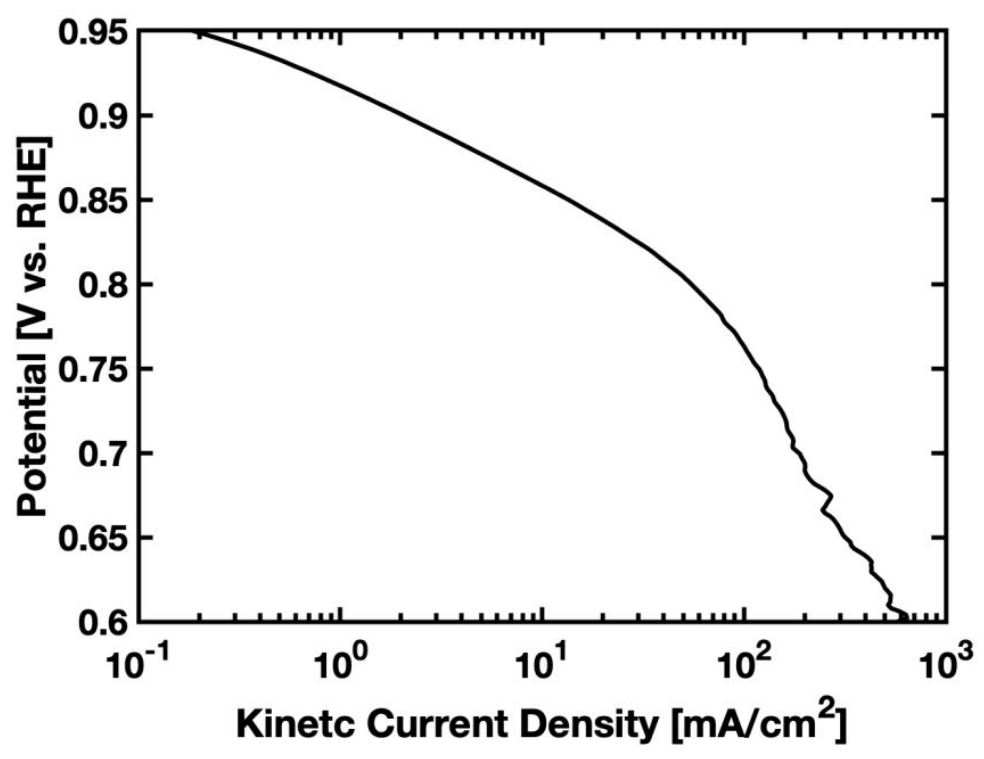


Figure S1: Tafel plot of the ORR current density for a Pt-poly electrode rotating at 1600 RPM in $\mathrm{O}_{2}$ saturated $0.1 \mathrm{M} \mathrm{NaOH}$. Currents are corrected for solution resistance loses and the capacitive current from the electrochemical double layer.

\section{Effectiveness Factor Model}

To simulate the mass transport limitations through the active catalyst layer supported on the rotating disk electrode, an effectiveness model was imposed on the Pt-poly kinetic data. In this way a continuous range of Thiele moduli can be added to a base case with a constant $100 \%$ effectiveness factor (fixed non-porous electrode) to simulate an active layer with diverse properties (thicknesses, porosities, conductivities, etc.).

The effectiveness factor model used is a modified model based on a macrohomogeneous system representative of a $\mathrm{RDE}$ from the literature ${ }^{2}$. The relevant equations are shown below:

$$
\begin{gathered}
\left.E F=\frac{\tanh \left(\phi_{T}\right)}{\phi_{T}} \quad \text { (Equation } \mathrm{S} 1\right) \\
\phi_{T}=\sqrt{\frac{\Gamma K \gamma L^{2}}{n F D_{c}} * \frac{1}{j_{0}} * j_{k}(p t)}
\end{gathered}
$$

Where $\mathrm{EF}$ is the effectiveness factor, $\phi_{T}$ is the Thiele modulus, $\Gamma$ is a temperature parameter, $\mathrm{K}$ is a lump kinetic term, $\gamma$ is the surface area to volume ratio of the pores, $\mathrm{L}$ is the film thickness, $\mathrm{n}$ is the number of electrons transferred, $\mathrm{F}$ is Faraday's constant, $\mathrm{D}_{\mathrm{c}}$ is the diffusion coefficient, $\mathrm{j}_{0}$ is the exchange current density and $j_{k}$ is the kinetic current density of the Pt-poly electrode. The values used for the constants are shown in Table 1. 
Table S1: parameters used in the Thiele modulus equation

\begin{tabular}{|c|c|}
\hline Parameter & Value [units] \\
\hline$\Gamma$ & 6 \\
\hline $\mathrm{K}$ & $12.2^{*} 10^{-6}\left[\mathrm{~A} / \mathrm{cm}^{2}\right]$ \\
\hline$\gamma$ & $0.0037\left[\mathrm{~cm}^{-1}\right]$ \\
\hline$L$ & varied $[\mathrm{cm}]$ \\
\hline $\mathrm{n}$ & 4 \\
\hline $\mathrm{D}_{\mathrm{c}}$ & $12^{*} 10^{12}\left[\mathrm{~mol}^{*} \mathrm{~cm}^{-1} \mathrm{~s}^{-1}\right]$ \\
\hline $\mathrm{j}_{0}$ & $2.4^{*} 10^{-7}\left[\mathrm{~A}^{*} \mathrm{~cm}^{-2}\right]$ \\
\hline
\end{tabular}

The overpotential loses through the active layer, which depend on the ionic conductivity of the film, was not included in our model. 


\section{References:}

1. Moniri, S., Van Cleve, T. \& Linic, S. Pitfalls and best practices in measurements of the electrochemical surface area of platinum-based nanostructured electro-catalysts. J. Catal. 345, $1-10$ (2017).

2. Gloaguen, F. \& Durand, R. Simulations of PEFC cathodes: an effectiveness factor approach.

J. Appl. Electrochem. 27, 1029-1035 (1997). 